\title{
Architectural hot-swap in NURBS surfaces: versioning of case studies, work in progress
}

\author{
A. Prichard-Schmitzberger \\ California State Polytechnic University, Pomona, USA
}

\begin{abstract}
This paper documents the testing of various applications of hot-swapping and precision modelling with minimal programming input reflecting the limited resources of small-scale practices. Hot-swapping identifies the principle of replacing components of a building during active design processes without altering its general appearance. The methodologies were tested through a series of courses on Digitally Enhanced Construction and Fabrication with architecture students at Calpoly Pomona. The teaching methodology allows not only the investigation of the required modelling accuracy but also the creation of prototypes and various versions of assembly alternatives. The paper covers the procedures developed to derive architectural form in a process of reverse engineering of specific architectural case. Flexibility or limitation of nonuniform surfaces for rapid prototyping and file-to-factory construction are investigated. Using versioning, a centralized design methodology with one source object for controlling and tracking 3d NURBS surfaces are detailed to material properties and using current building material which allows the implementation of pre-fabrication as well as mass-customization. Resulting projects allow assessment of time-consumption in the design-process; deviation of the proposed result from the original; overall complexity of assembly method; customization amounts of individual parts and manual effort. The procedures outlined in this paper would enable small architectural offices to enter competition with larger offices through integrating positive aspects of hotswapping in conjunction with prototyping without the typically necessary technical and fiscal casts.
\end{abstract}

Keywords: hot-swapping in architecture, reverse engineering, versioning, computer integrated construction and manufacturing, design processes. 


\section{Introduction}

The course Digitally Enhanced Construction and Fabrication establishes first contact with so-called "file to factory" [2] scenarios and rapid prototyping in architecture. A major aspect of the course, however, focuses on the practical application and the supposed seamless exchange of digital information in $3 \mathrm{~d}$ modelling, simulating a typical small-scale practice. Small-scale offices experience on one hand the financial strain of software and equipment costs and on the other hand affordability of qualified employees to be in competing range with larger office structures.

\section{Objective}

The advance of BIM - Building Information Modelling - and the integration thereof in the curriculum for accreditation purposes offers another challenge to digital architecture and its educators. BIM is associated with the relatively 'smart' structure of current software packages, among them so-called parametric modelers such as Autocad Revit and Archicad. However, these particular programs differ in application from potential software packages such as Generative Components, running on Microstation platforms, due to the fact that the major input follows industry standards rather than input derived from a designed architectural model, enabling definition and specification of customized detailing. Hot-swapping, a term borrowed from computer hardware, describes a potential method which allows a) quick development of a base $3 \mathrm{~d}$ model with enough flexibility and accuracy for future manipulation and b) exchange of construction methods up through a late stage of design and production.

\subsection{Course setup}

The initial 10 week, 3 unit course layout encompasses a lecture portion with a brief introduction to the history of prototyping and numeric machining. Differentiation between polygonal, Boolean and NURBS modelling is necessary to establish an understanding for the advantages and disadvantages in creation processes. NURBS models offer mostly more flexibility due to their parametric setup. Polygonal mesh models represent complex structures themselves and are therefore undesired. Much of contemporary architecture shows intention and interest in generating quasi-automatic forms and structures, genetic algorithms, and parametric design methodology. They engage programming/scripting, setting inputs, initial conditions, controls, and performance, all of which are criteria to evolve a form or structure or to produce a "population" of possible architectural phenotypes which are then selected according to extrinsic criteria or self-referencing elaborated. Manuel DeLanda once characterized this shift towards the genetic algorithm as the third wave of digital modelling software, one which would finally supplant all the previous identities of "designer" and intentionality in favor of diagrammatic processes [3]. This process is mostly 
formal and despite its aspiration a very often pseudo-scientific approach that appears removed from any material aspects. Hot-swapping follows a different premise.

\subsubsection{Hot-swapping configuration}

Hot-swapping as a technique is detached from the architectural discussion on form and formal tendencies. The re-association with fabrication, construction and affiliated practices is tied to the computational procedures. The digital model serves as surface model and offers the conditions upon which material properties and structural methods can be exchanged in a timely fashion without the need for re-creating the geometries. In architectural education the tools are limited, and the understanding of architectural assembly is still a traditional one. Architectural construction methods are sequenced in accordance with the history of modernist, post industrial-revolution production, choosing pre-arranged certified techniques and using discrete building elements to form a hierarchical build-up sequence. These independent elements represent a simple nested hierarchy, easy to control in assembly and for its assembly schedule.

\subsubsection{File-to-factory}

File-to-factory scenarios and the proclamation "one-building - one detail" [4] by $\mathrm{K}$. Oosterhuis of ONL require a new production setting, reduction of material and the generation of assemblies at a pre-construction phase as well as a different structural fabrication scenario. While bypassing the rather linear, formgenerating relationship of architecture and technology, mostly represented through the software and its commanding algorithms, he further re-introduces the relevance of practical application, looking for a new position in the construction process. Utilizing the digital model not only for representational, diagrammatic or conditional purposes, but also for applying an entire material and assembly process as well as documentation method is core to Building Information Modelling. However closely related, his practical application does not submit to standardized detailing and building methods but offers via 'hot-swapping' and parametric design the ability to control the production process in conjunction with the digital model until a very late stage in the design-to-production process $[5]$.

\subsection{Design methodology}

Throughout studies it appears that for an applicable generation of conditions which should allow a hot-swap of features, a parametric surface model provides the highest flexibility in a) establishing a controllable form and b) continually applying detailing features. For this investigation it is necessary that current discussions concerning performative aspects of surfaces strictly follow the practical application only to avoid unnecessary distractions from the objected goal. A reversed, traditional approach of developing a structural grid and forming the architecture after its constraints would limit any flexibility in the exchange of generation methods. Any parametric surface can be treated as a field 
and therefore subdivided into elements, following a process similar to tessellation of digital surfaces first-hand. However, a structurally correct, generative tessellation would again require specific scripting effort. The engineering-design application mainly focuses on a realistic structural composite solution at this point.

The maintenance of a relatively simple NURBS surface in Rhino3D offers a highly flexible base for any further manipulation, since the parametric values are reduced to few control-points in contrast to polygonal meshes, with a comparatively excessive amount of manipulation vertices. Structural application can be simply pulled, projected onto the surface or created via isocurves, fig. 1, and despite causing occasional undesirable, uncontrolled effects in varying degrees of the surface curvatures, the results remain largely flexible for manipulation. Further detailing is has a high level of time consumption due to the manual aspect of modelling; each elemental intersection is revisited with each alteration of the conditions and if necessarily corrected through either Boolean operations or remodelling.

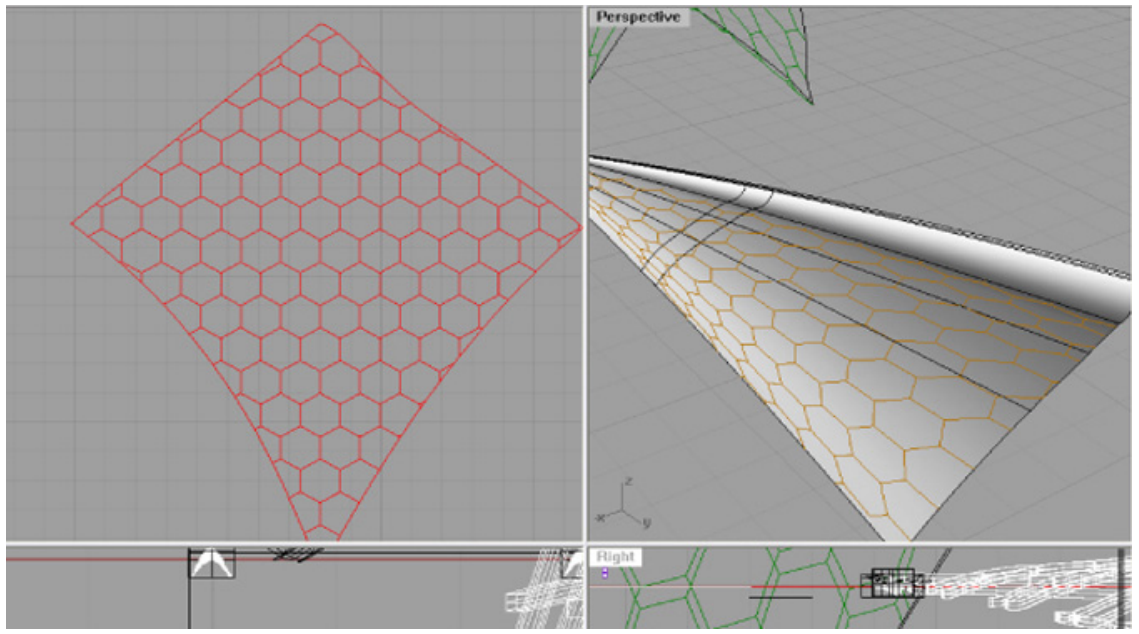

Figure 1: Example for pulling structure grids to a surface. (Student: D. Rogers).

\section{Practical application}

Given practical limitations the course setting intentionally abandons the introduction of scripting to investigate and manage architectural data in a more traditional sense. The importance of programming and respectively scripting is undisputed; programmability is a unique property of the digital medium. Design methodology might be opposed by the exactness inherent to programming itself; though this concern was not reflected by the objectives of this course. 


\subsection{Concrete modelling}

The case-studies chosen for reverse engineering are F. Candela's Restaurant Mantiales in Xochimilco, Mexico, built in 1958-59, and the 1959 version of F. Kiesler's Endless House. Candela's Restaurant Mantiales represents an elaborate thin concrete shell structure; the main form, a rotated Hypar hyperbolic paraboloid (a non-planar surface in which at any point are two intersecting straight lines) represents a challenge in reconstruction itself without the support of a mathematical formula; in addition its non-conventional selfsupporting structure ultimately removes it from the typical possibilities to be developed under use of regular BIM approaches. Similarly, Kiesler's Endless House follows in its bio-morphic structure a non-regular architectural form, which demands not only strategic modelling consideration but also understanding of complex geometries in space. Both models were reduced to slightly simpler geometries in order to allow adequate time-management of the course, fig. 2 .

\subsubsection{Reverse engineering}

In order to offer best resolution in the surveillance practice, Reverse Engineering (RE) is brought into consideration. RE is a process describing recovery or discovery of technological principles of a mechanical, or in this case architectural, application through analysis of its structure, function and operation, in architecture becomes comparable to a detailed case study. An architectural example can be evaluated and understood through its geometric, structural and technological assets. The procedure further enables understanding of core-principles of generating ruled surfaces and their flexibility as well as applying those principles to fixed parameters derived from the studied object.

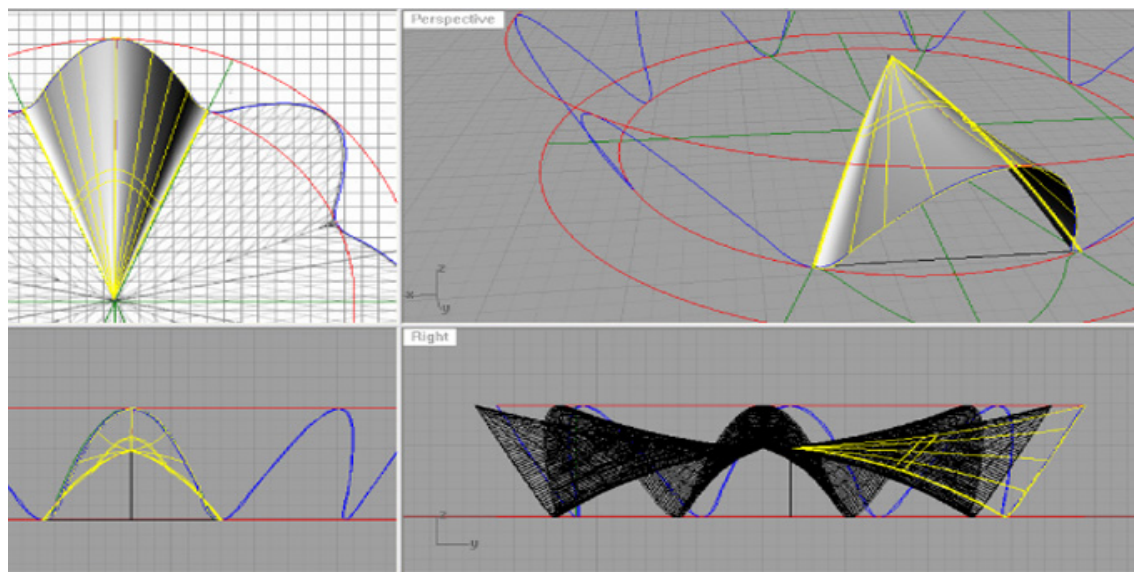

Figure 2: Deriving the Hypar from defining geometries. (Student: D. Rogers). 


\subsubsection{Versioning}

After assessment of scope and modelling technique the principle of versioning is introduced and applied in parts. Versioning describes a centralized design methodology with one source object for controlling and tracking and in chosen case studies students are assigned one successfully developed model to generate individual considerations of construction method.

\subsection{Implementation}

Adopting the traditional method of tracing a digital line-drawing of the original architecture, a set of B-Splines and resulting 1/8 segments of NURBS surfaces for the Mantiales model are produced. To reconnect those after the rotational array is itself a transitional step, non-regular surfaces need to be inserted which transform a single a surface ultimately into a polysurface, reducing the flexibility of the system as a whole. Afterwards a cleaner surface can be derived through a lofting procedure from extracted sections and edges, fig. 3 .

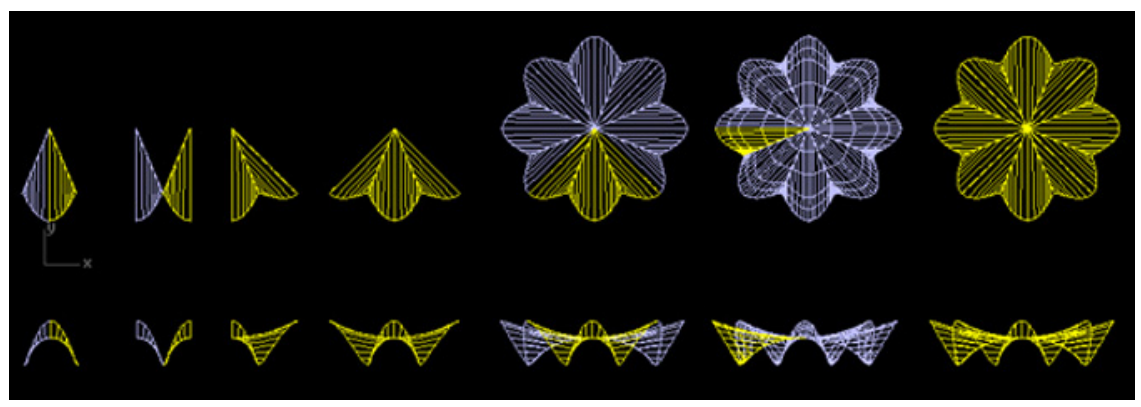

Figure 3: Completing the full rotational structure for the Restaurant Mantiales (Student: M. Gonzales).

In the case of Kiesler's Endless house the geometry is far more complex, tracing efforts to develop boundary curvatures and respectively cross-sectional shapes demand advanced modelling skills. A lofting method of interpolating equally spaced cross sections within the boundary trace-lines for individual pods provides satisfying results. A reduced geometric model of the exterior envelope of Kiesler's project is developed. The blending of the pod-shapes to one joint shape adds a level of imprecision and deprives the NURBS model of its initial flexibility, fig. 3. Full parametric modelling as offered through Microstation GC might deliver a better, faster product; in current version of the House the time consumption is demanding to fall back on available tools with steep learning curve such as Rhino3D, fig. 4.

In both Kiesler's Endless House and Candela's Restaurant Mantiales, various approaches in different complexities were repeatedly re-drawn to optimize the base model; these models received further manipulation through individual interpretations and considerations of structural systems through projections onto the surfaces themselves. This initial projections already allow first analysis and 
re-application of different structural systems, which then, through simple sequenced processes of lofting and extruding lead to rudimentary but applicable structural solutions, figs. 5(a), (b).

Any additional, engineered input from this point on illustrates the limitations of modelling without scripted environments. Despite the maintained precision, manual input of eventually corrected, structural or material assumptions would require re-adoption in a manual, linear fashion and therefore would add up to potential human error or imprecision as well as consumed time.
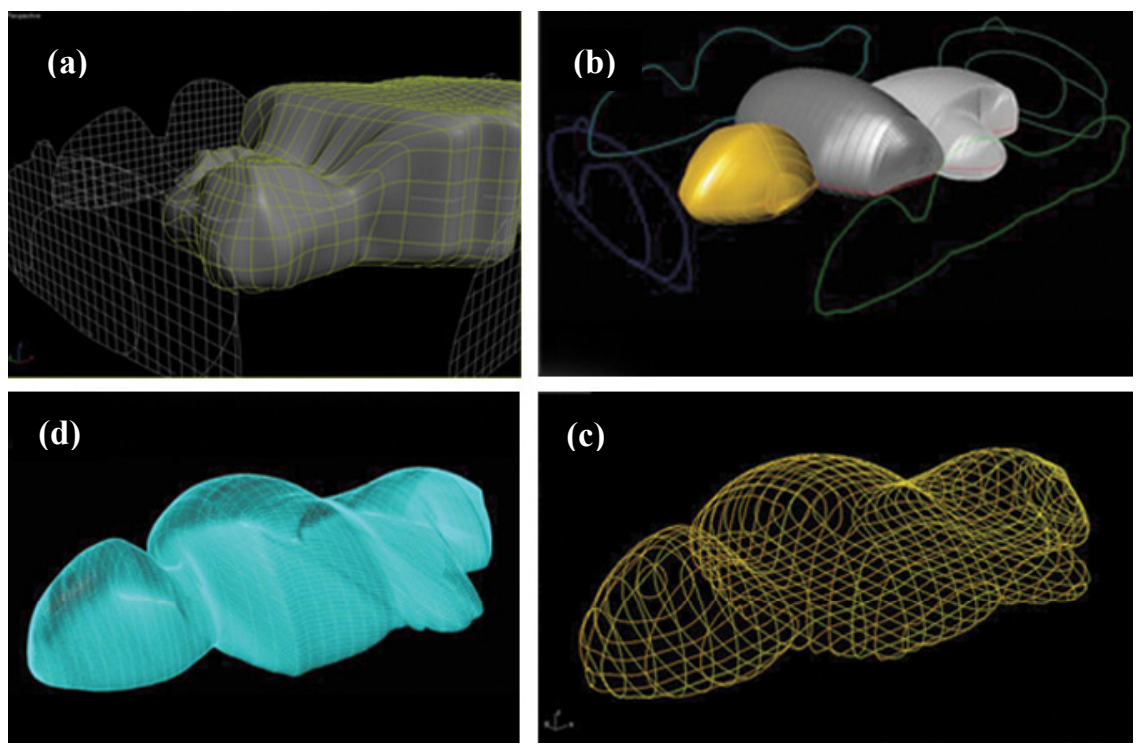

Figure 4: Development of Kiesler's surface model (a) mesh transformation delivers uncontrollable surface, (b) pod-method generates more accurate solution, (c) pods are merged, (d) an isoparm curve skeleton is derived) (Student: J. Cabreras).

\subsection{Results}

Results vary in quality, but demonstrate initial success of the conceptual state of hot-swapping method in architectural design. The ability to offer a multiplicity of solutions - versioning - in a relatively short time allows affability in material and engineering negotiation, fig. 6.

\section{Conclusion: programmer - an architectural specialization}

Schedule, availability of technical support and architectural challenge remain key-factors for competition of small architectural practices to large offices; displayed approach demonstrates that with some geometric constraints the small 


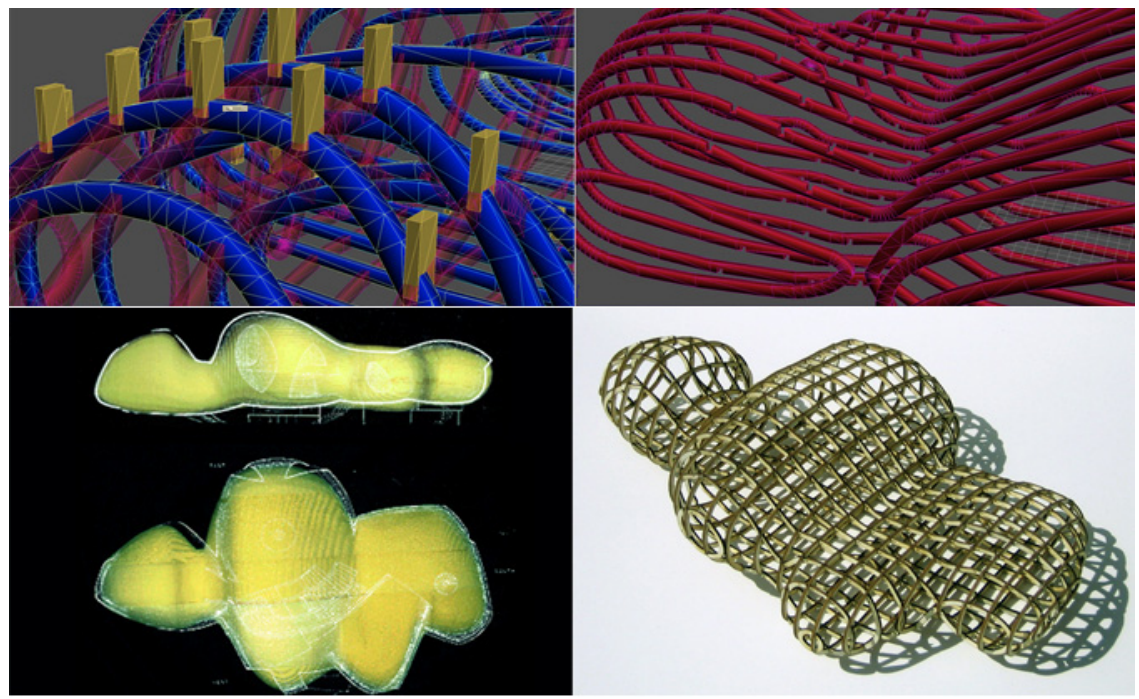

(a)
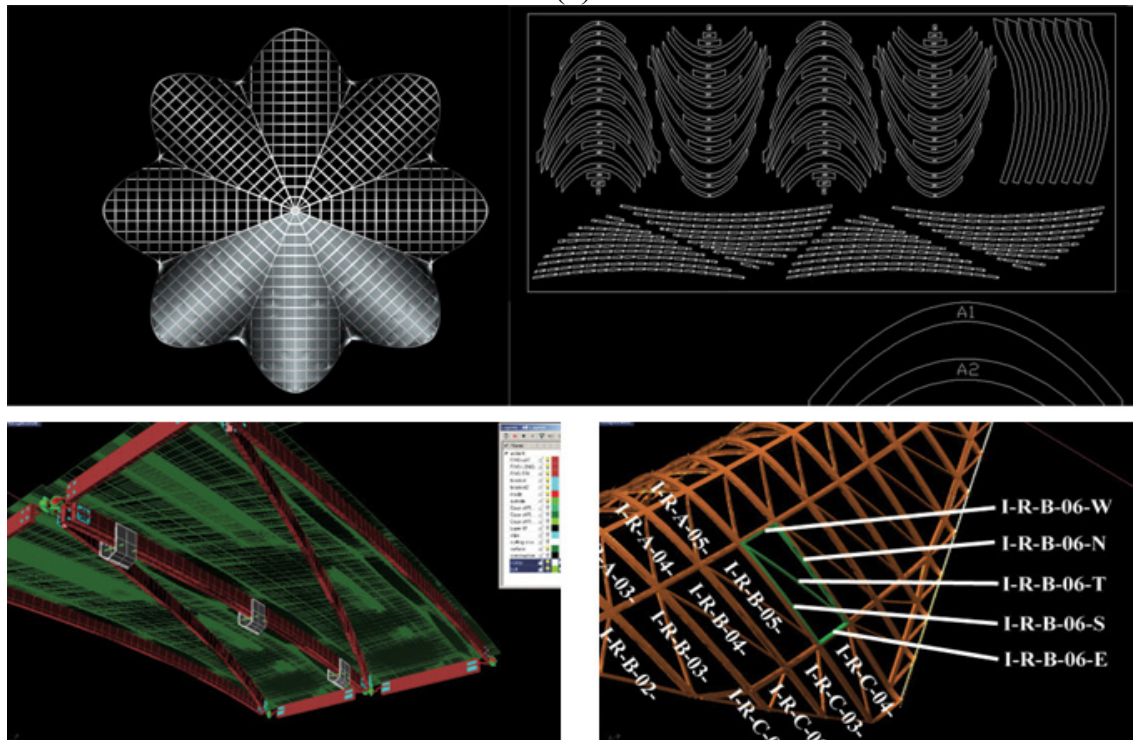

(b)

Figure 5: (a) Clockwise: (i), (ii) intersections between crossing members are derived manually, a time-consuming process, lacking specific accuracy, (iii) CNC-prototype underplayed the original drawing and (iv) laser-cut structural model (production time incl. file preparation 50 hours) (Students: J. Leong, E. Liu, H. Ming, A. Hernandez); (b) Clockwise (i), (ii) finalization for production, creation of cut-sheets for laser-cutting (student: M. Gonzales), (iii), (iv) Tessellation approach and detailing including tagging of different elements (Student: E. Scott.) 


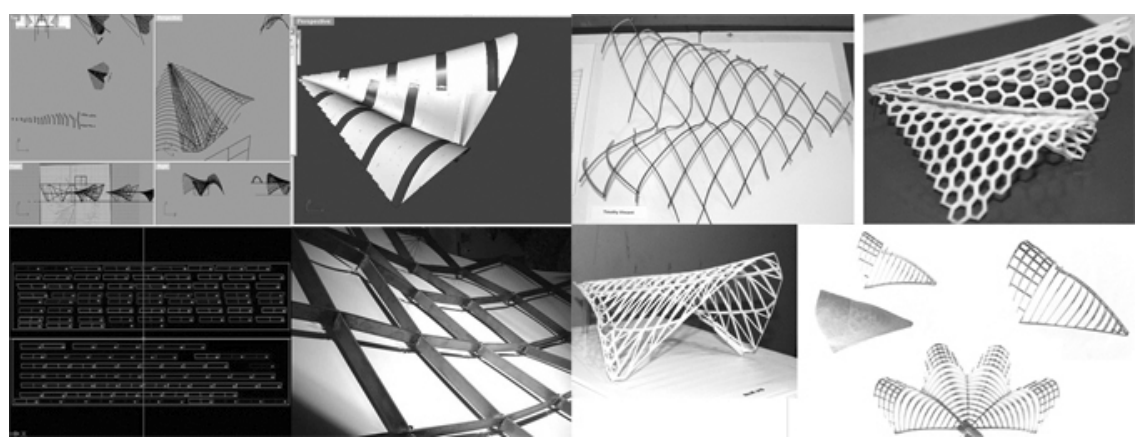

Figure 6: Clockwise from top left: different approaches with different Prototypes, shingled surface model with diagonally braced sheet metal frame (Student: T. Vincent), hexagonal structure (Student D. Rogers), cut-sheet for welding mock-up (Student T. Vincent), tessellation model (Student E. Scott) and grid-lattice (Student M. Gonzales).

office is in competition with the traditional large office. However, hot-swapping as technique remains, despite improvements in modelling skills, modelling accuracy and software advancement, a prerogative of designers with adequate programming skills and education. Real-time events, as Axel Kilian points out, require programming of these events so that the computational event takes place during its calculated execution [6]. Common affordable and available software packages currently does not provide this commodity in other form than indigenous scripting language; scripting itself supports customization of software which does not inherently support enough flexibility in its command structure and can therefore be seen as a reverse approach itself. Software designed for architects seems to gear more towards implementing industry standards rather than generating a desirable tool for architectural design. Education itself and its accreditation systems abandon vital elements for at least allowing students to customize the appropriated software; neither descriptive geometry nor programming skills are enforced. Therefore educators are forced to rather comply with standards initiated by software producers and industry, than to represent a contributing element to the industry itself.

Respectively, a small practice cannot currently take full advantage of new trends in architecture, due to the budget and schedule challenges for employment of specialists and the rigid hierarchy in construction business. Given the current climate of academic education in architecture programs and the lack of substantial pre-education in computing skills, the course is partially used to examine success and failure of the system in parts. Building Information Modelling appears to offer desirable appeal to such structures, relying on the safe implementation of established construction methods and materials. Alternative routes, as demonstrated in examples by practices of Frank O. Gehry Associates and ONL become showcases and demonstrate the position of corporation versus academia; academia is currently coerced to follow the lead of few successful 
large practices an their techniques. Knowledge and understanding of engineering, industrial production sequences, explicit computational and geometric training simplify the architectural and respectively digital production process and would further allow architectural academia structures to regain its position as informant educator.

\section{References}

[1] Supported by a grant from the College of Environmental Design, California State Polytechnic University.

[2] Oosterhuis K, The Architect's new Data-Driven Practice (Chapter 3). Hyperbodies, Towards an E-motive Architecture, Birkhaeuser: Basel, Boston, Berlin, pp7-10, 2003.

[3] De Landa, M.1, Philosophies of Design, The Case of Modelling Software, Verb Processing, Actar: Barcelona, 2001.

[4] Oosterhuis, K., Building Bodies (chapter 7), Hyperbodies, Towards an Emotive Architecture, Birkhaeuser: Basel, Boston, Berlin, pp 17-22,2003.

[5] Oosterhuis, K., Bier, H. Aalbers, C., Boer, S., Programmable Architecture, Proc. of ACADIA 23, Fabrication, pp 3-5, Cambridge and Toronto, Ontario, Canada, 2004.

[6] Kilian, A., The Importance of Programming, Defining Digital Space Through a Visual Language, p 13, Master of Sciences Thesis, Massachusetts Institute of Technology, Department of Architecture, 2000, http://destech.mit.edu/akilian/newscreens/thesis2000/pdfversion.PDF 